\title{
In vitro modeling of HER2-targeting therapy in disseminated prostate cancer
}

\author{
JENNIE ANDERSSON* ${ }^{*}$, MARIA ROSESTEDT ${ }^{*}$, VERONIKA ASPLUND, NAZILA YAVARI and ANNA ORLOVA \\ Preclinical PET Platform, Uppsala University, Uppsala, Sweden
}

Received June 11, 2014; Accepted July 25, 2014

DOI: 10.3892/ijo.2014.2628

\begin{abstract}
Prostate cancer (PCa) is the most common cancer type among men. Treatments against advanced $\mathrm{PCa}$ are limited and in many cases only palliative. In a later, androgent independent, stage of PCa androgen receptors can be activated without interaction with ligand, i.e., by receptors of tyrosine kinase (RTK) family in the outlaw pathway. Human epidermal growth factor receptors HER 2 and EGFR belong to RTK-family. HER2 is one of the main actors in the outlaw pathway with EGFR as the preferable heterodimerizing partner. We hypothesized that information on HER2 expression in advanced PCa could be useful for selection of patients for anti-RTK therapy and monitoring of therapy response. A panel of PCa cell lines (LNCap, PC3, DU-145) was subjected to a 8-week treatment using drugs influencing the RTK: trastuzumab (anti-HER2), 17-DMAG (Hsp90 inhibitor), alone or in combination, and their HER2 and EGFR expressions were compared with non-treated cells. Treatment with trastuzumab decreased proliferation of LNCap and DU-145 cell lines, while 17-DMAG and trastuzumab/17-DMAG combination affected all three cell lines. HER2 expression was significantly increased in PC3 cells, the most resistant cell line. On the contrary, in responding cells (LNCap and DU-145) HER2 expression decreased, accompanied by increased EGFR expression. However, additional treatment of cells with cetuximab (anti-EGFR) did not give any additive effect to trastuzumab. In this study the response to anti-RTK therapy proved to vary between different PCa cell lines. We have demonstrated that RTK targeting treatments may affect the phenotypic profile of PCa tumor cells that correlates with therapy outcome. Observation of such changes during treat-
\end{abstract}

Correspondence to: Dr Maria Rosestedt, Preclinical PET Platform, Uppsala University, Dag Hammarskjöldsv 14C, 3 tr, 75183 Uppsala, Sweden

E-mail: maria.rosestedt@pet.medchem.uu.se

*Contributed equally

Key words: prostate cancer, human epidermal growth factor receptor type 2 , targeted therapy, molecular imaging, in vitro modeling ment could be used for monitoring and an improved therapy outcome.

\section{Introduction}

Prostate cancer ( $\mathrm{PCa}$ ) belongs to the most heterogeneous malignant tumors, both histologically and clinically. Initially $\mathrm{PCa}$ is slow growing, confined to the prostatic gland and dependent on testosterone for proliferation. Treatment is not always necessary and in many cases the only course of action is simply referred to as watchful observation. About a third of the patients on watchful observation starts an active treatment within their first 5-years post diagnosis $(1,2)$, and the younger men (55-69 years) gain more from active screening and treatment $(3,4)$. However, in patients receiving first line therapy a recurrent and more aggressive type of $\mathrm{PCa}$ occur in $20-40 \%$ of cases within 10 years (5). At this point, the tumor can start to invade the tissues surrounding the prostate gland, and the situation requires a more aggressive treatment, preferably a combination of androgen ablation and local irradiation therapy (6).

Although the combinational therapy can induce a clinically stable state for 1.5-3 years, the absence of androgen and the heterogeneity of the tumor can lead to a clonal selection of androgen independent cells. Androgen-independent PCa cells can proliferate and survive in the absence of androgen, also they are less susceptible to the growth suppressing properties of androgen ablation. Hence, the recurring PCa is typically androgen-independent, more likely to metastasize, less responsive to available treatments and give patients a median survival of 12-24 months, depending on applied treatment (7,8). Previously, 6 different molecular mechanisms have been identified as being involved in the development of androgen-independence; hypersensitive pathway, promiscuous pathway, outlaw pathway, bypass pathway, balance between coactivators and corepressors and PCa stem cell regeneration (9). This study focuses on the outlaw pathway which has the capacity to activate androgen receptor signalling by ligand-independent mechanisms involving receptor tyrosine kinase (RTK) (10-12).

Human epidermal growth factor receptor type 2 (HER2) is one of the actors of the outlaw pathway, and its expression was found in about $20 \%$ of analyzed PCa samples in a study involving 2,000 samples, both primary tumors and metastases (13). In androgen diminished environment, 
HER2 promotes cell survival, suppresses apoptosis and increases cell motility, hence being strongly associated with a more advanced disease and tumor stage $(13,14)$. This enables a selective survival for HER2 expressing cells leading to an accelerated tumor progression and androgen-independence causing an increased resistance to existing therapy $(15,16)$. Epidermal growth factor receptor (EGFR) is another member of HER-family and also plays a role in the outlaw pathway. Since HER2 lacks a natural ligand, it needs to dimerize with another family member to become active, and its preferable partner is EGFR. Upregulation of HER 2 and EGFR is known to be an important aspect in the development of different types of cancer (17).

In this study, we therefore speculate that HER2 could be a suitable target for treatment of advanced PCa. Several therapeutics suppressing HER2 activity in cancer cells are now in clinical use $(18,19)$. In vivo molecular imaging of HER2 expression in advanced PCa could stratify patients to anti-HER 2 therapy and further monitor therapy outcome (20). Trastuzumab (Herceptin) is an approved therapeutic monoclonal antibody that binds to the extracellular domain of HER2 and downregulates its expression (21). The drug is today in clinical use for treatment of HER 2 expressing breast cancer (BCa) and studies including the use of trastuzumab in endometrial (22), peritoneal, pancreatic, ovarian, stomach (23) and bladder (24) tumors are ongoing. Heat shock protein 90 (Hsp90) is a molecular chaperon essential for the stability and proper folding of proteins involved in e.g. signal transduction pathways and cell growth. The expression of Hsp90 in cancer cells is 10-fold higher than in normal cells, and it is involved in multiple steps of tumor progression, including the maintenance of HER2. The drug 17-DMAG (17-(dimethyl-aminoethylamino)-17-demethoxygeldanamycin) is a water-soluble Hsp90 inhibitor which prevents the intracellular production of HER2 and simultaneously disrupts multiple signalling pathways (25). Cetuximab (Erbitux) is a monoclonal antibody which is today used in treatment of EGFR expressing colorectal cancer. The drug binds to the extracellular domain of EGFR and blocks receptor dimerization and activation, hence promoting receptor downregulation (26). The aim of this study was to investigate the effect of different RTK-targeting therapy regimes on survival of PCa cells in vitro. We also hypothesised that therapy response could correlate with changes in RTK membranous expression and could be used for therapy monitoring.

Three PCa cell lines with different properties were selected for this study: LNCap (lymph node metastasis of $\mathrm{PCa}$, androgen and estrogen receptor positive), PC3 (bone metastasis of $\mathrm{PCa}$, androgen sensitive), and DU-145 (brain metastasis of PCa, hormone insensitive). Together these cell lines might reflect tumor heterogeneity. Membranous expression of HER 2 and EGFR in this cell lines was documented earlier $(27,28)$. Cells were treated weekly with drugs influencing HER2 and EGFR expression. Membranous expression of HER2 and EGFR as well as cell survival in response to therapy was studied. Trastuzumab and cetuximab which binds to the extracellular domain of HER2 or EGFR, respectively, and downregulates their expression, and 17-DMAG Hsp90 inhibitor, were selected as drugs for this study.

\section{Materials and methods}

The cell lines LNCap, PC3 and DU-145 from the American Type Culture Collection were provided by LGC Standards. The cell lines were evaluated for HER2 and EGFR expression earlier $(27,28)$. Cells were cultivated in complete RPMI-media, supplemented with $10 \%$ fetal bovine serum (FBS), $2 \mathrm{mM}$ L-glutamate, $100 \mathrm{IU} / \mathrm{ml}$ penicillin and $100 \mu \mathrm{g} / \mathrm{ml}$ of streptomycin. Media for LNCap cells were also supplemented with Na-pyruvate and HEPES. All reagents including media and trypsin-EDTA were from Biochrom AG, except Na-pyruvate (Lonza, BioWhittaker). All plastics for cell culturing were from Corning, and the cells were cultivated in a humidified incubator with $5 \% \mathrm{CO}_{2}$ at $37^{\circ} \mathrm{C}$.

For in vitro treatment trastuzumab (Herceptin, infusion $21 \mathrm{mg} / \mathrm{ml}$, Roche), cetuximab (Erbitux, infusion $5 \mathrm{mg} / \mathrm{ml}$, Merck Serono), and 17-DMAG (Calbiochem) were used.

For HER2 quantification affibody molecule $\mathrm{Z}_{\text {HER2:2395 }}$ (Affibody AB) was used, and for EGFR-cetuximab coupled to CHX-A"DTPA chelator ([(R)-2-Amino-3-(4-isothiocyanatophenyl)propyl]-trans-(S,S)-cyclohexane-1,2-diamine-pentaacetic acid Macrocyclics). Radiolabeling of $Z_{2395}$ with technetium- $99 \mathrm{~m}$ was performed using the protocol described by Ahlgren et al (29). Cetuximab was labeled with indium-111 according to the method described earlier (28).

Radioactivity was measured with an automated gamma-counter with 3-inch NaI(Tl) detector (1480 Wizard, Wallac Oy). Cells were counted using electronic Scepter ${ }^{\mathrm{TM}}$ cell counter from Millipore.

Student's t-test was used to evaluate the significance in changes of proliferation and receptor expression. P-value below 0.05 was considered as significant (indicated by the star symbol in respective graphs).

Targeted treatment protocol. The cells were treated weekly according to the following protocol: cells were seeded at $10^{5}$ cells/culture flask and next day subjected to a regimen of trastuzumab, 17-DMAG, cetuximab or different combinations (trastuzumab/17-DMAG, trastuzumab/cetuximab). One group was used as a control and treated in the same manner as all other cells, but not subjected to any drugs. The same procedure was repeated weekly. For all treatments the drugs were diluted in cell cultivation media to: $0.05 \mathrm{mg} / \mathrm{ml}$ trastuzumab, $0.03 \mathrm{mg} / \mathrm{ml}$ cetuximab and $0.003 \mu \mathrm{g} / \mathrm{ml}$ 17-DMAG. Receptor quantification was done for groups treated with trastuzumab, 17-DMAG and trastuzumab/17-DMAG at the termination of the experiment (after 8 weeks of treatment). All experiments were performed in quadruplicates.

Receptor quantification. Cells were seeded at $10^{6}$ cells/ dish $24 \mathrm{~h}$ prior to the experiments. On the day of the experiment an excess amount of unlabeled $\mathrm{Z}_{2395}(60 \mathrm{nM}$, for quantification of HER2) or cetuximab (100 nM, for quantification of EGFR) was added to half of the wells and the cells were left to incubate for $1 \mathrm{~h}$ at room temperature (RT), followed by addition of $0.6 \mathrm{nM}{ }^{99} \mathrm{~m} T \mathrm{Tc}$-labeled $\mathrm{Z}_{2395}$ or $10 \mathrm{nM}{ }^{111} \mathrm{In}$-labeled cetuximab to all wells and $1 \mathrm{~h}$ of incubation at RT. The cells were then trypsinized, resuspended, collected and counted according to protocols described earlier $(27,28)$. The radioactivity in cell samples 

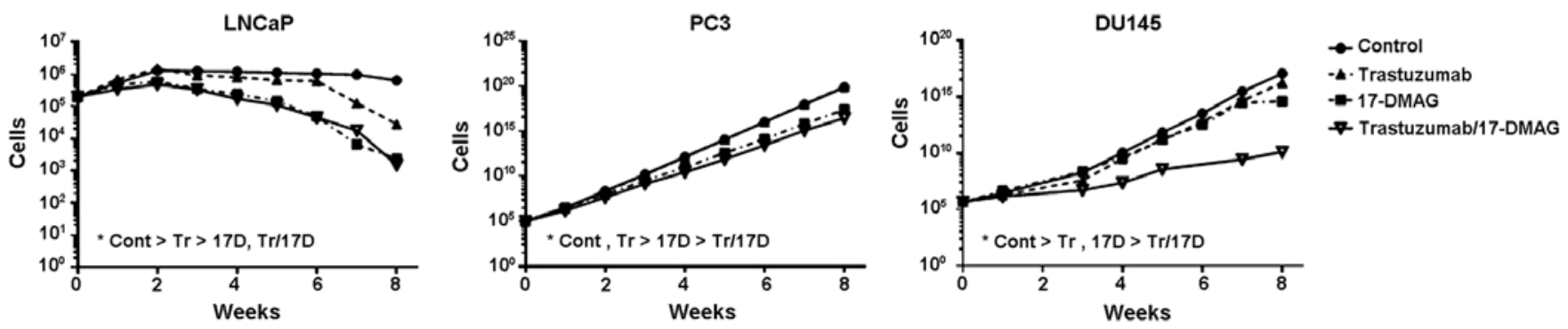

Figure 1. Growth curves for LNCap, PC3 and DU-145 cells. Cells received a weekly dose of $0.05 \mathrm{mg} / \mathrm{ml}$ trastuzumab, $0.003 \mu \mathrm{g} / \mathrm{ml} 17$-DMAG or a combination of the two. A control group was consistently kept in the same conditions, but not subjected to any treatments. Error bars not visible.
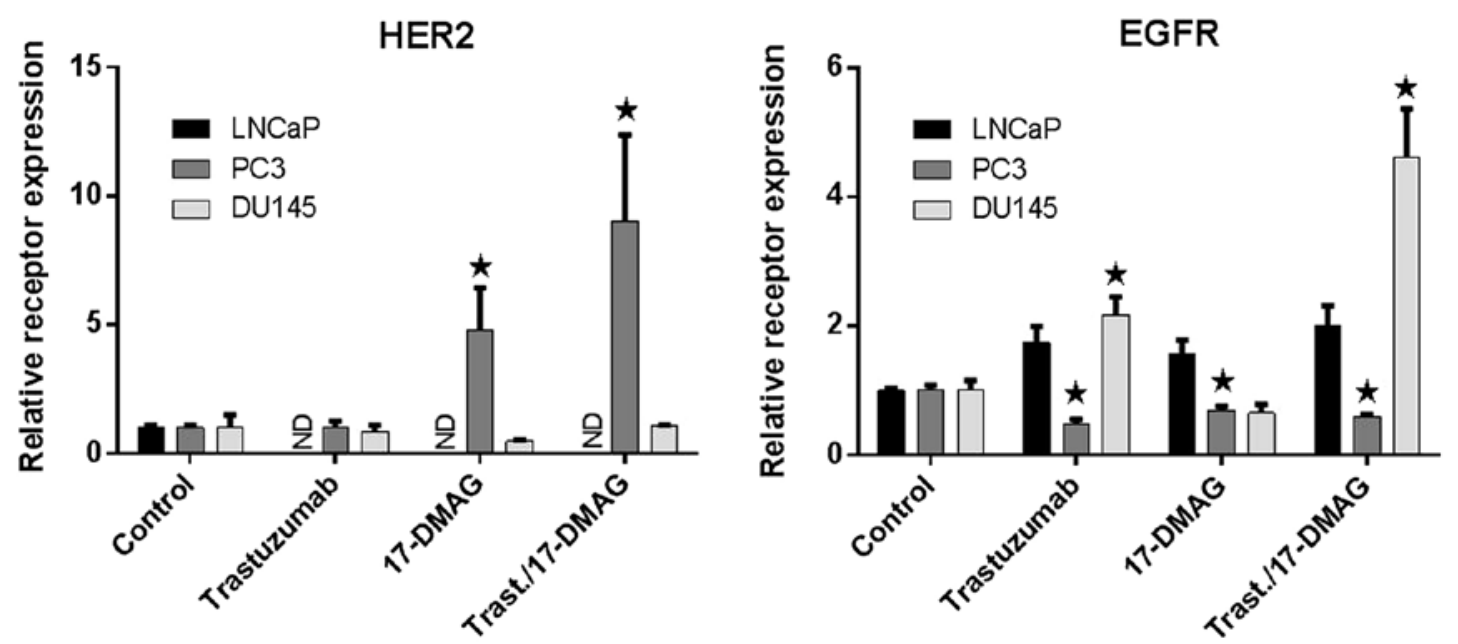

Figure 2. Receptor expression post-treatment. Graph showing changes in HER2 and EGFR expression after long-term weekly treatment with trastuzumab, 17-DMAG or a combination of the two in PCa cell panel. HER2 expression was not detectable in LNCap cells receiving treatment. Significance is indicated in the graph with stars. ND, HER2 expression not detectable in LNCap cells.

was measured in an automated gamma-counter. All experiments were performed in triplicates.

\section{Results}

By subjecting cells to a long-term treatment consisting of sole or combinational drug administration (Fig. 1) we studied the proliferation inhibitory response. Treated cells varied very much in response depending on treatment. LNCap cells were not only the most sensitive to all treatment but also demonstrated inhibited proliferation in control group, probably due to treatment protocol.

The HER2 targeting treatment with trastuzumab demonstrated a significant decrease in proliferation for LNCap and DU-145 cells, whereas the drug showed a rather proproliferative effect in PC3 cells. Administration of sole Hsp90 inhibitor 17-DMAG resulted in a significant growth inhibitory effect in all tested cell lines. Combination therapy using trastuzumab/17-DMAG did not contribute with any additive growth inhibitory effect in LNCap cells over sole 17-DMAG; but resulted in an induced growth inhibition effect for both PC3 and DU-145 cells.

After 8 weeks of treatment, EGFR and HER2 membranous receptor expression in LNCap, PC3 and DU-145 cells (Fig. 2) were analyzed to evaluate the response to long-term treatment with trastuzumab, 17-DMAG, and their combination. As response to all subjected treatments, EGFR expression in LNCap cells was upregulated: receptor expression levels were similar for cells subjected to single trastuzumab or 17-DMAG treatment, however, the combination of trastuzumab and 17-DMAG resulted in a 2 -fold increase as compared to untreated cells. The HER2 expression levels were non-detectable in LNCap cells exposed to drugs. EGFR receptor quantification in PC3 showed a significant, but rather small, downregulation in expression as a response to trastuzumab, 17-DMAG and their combination. On the contrary HER2 expression was upregulated in PC3; 4-fold increase as a response to 17-DMAG treatment alone, whereas the addition of trastuzumab to 17-DMAG resulted in a significant 8-fold increase. In DU-145 cells, a significantly increased expression of EGFR could be observed for trastuzumab treatment and a significant 3.5-fold increase for the combination of trastuzumab and 17-DMAG. However, EGFR expression was not influenced by 17-DMAG sole treatment. HER2 membranous expression in DU-145 cells was not changed in response to any tested therapies.

Because of the increased EGFR expression previously demonstrated in $\mathrm{PCa}$ cells as response to trastuzumab/ 17-DMAG treatment, the cell panel was long-term exposed 

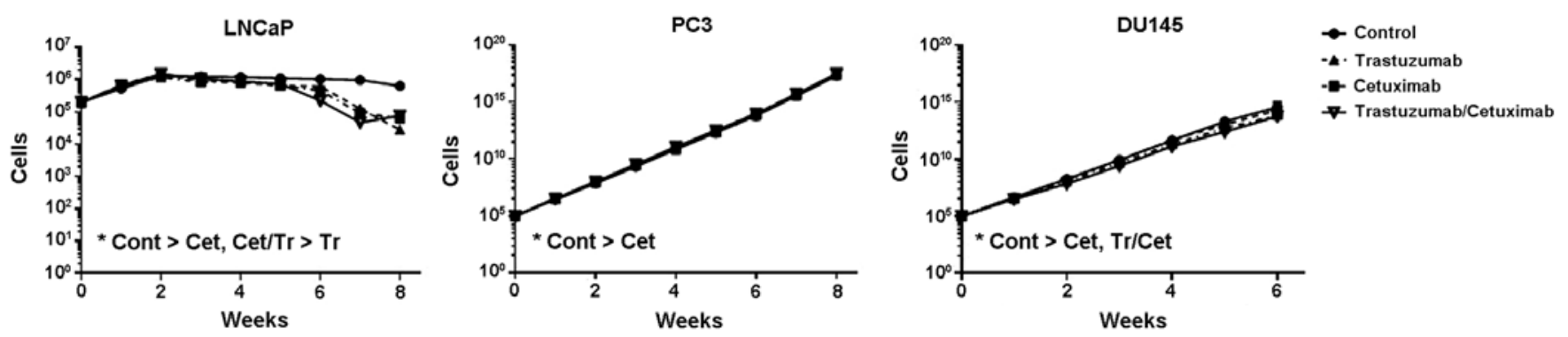

Figure 3. Growth curves of LNCap, PC3 and DU-145. The cells were treated weekly with a dose of $0.05 \mathrm{mg} / \mathrm{ml}$ trastuzumab, $0.03 \mathrm{mg} / \mathrm{ml}$ cetuximab or a combination of the two. A control group was consistently kept in the same conditions, but not subjected to any treatments. Error bars not visible.

to sole cetuximab treatment, or to a trastuzumab/cetuximab combination. LNCap cells treated with sole cetuximab demonstrated a reduced cell proliferation in comparison to control cells (Fig. 3). PC3 cells were not affected by cetuximab treatment, whereas DU-145 cells demonstrated a minor decrease in cell growth. The addition of trastuzumab showed no or minor effect in tested cells.

\section{Discussion}

HER2 is an RTK which is overexpressed in different cancer types and is involved in cell division, increased cell motility and suppression of apoptosis. Previous studies $(13,30)$ have reported HER2 expression in $\mathrm{PCa}$ as well as its role in the progression of androgen-independent $\mathrm{PCa}$ (31). HER2 is therefore considered as a valid target for treatment of advanced PCa. For this study, we hypothesized that monitoring of HER2 expression as response to sole or combinatorial RTK-targeting therapy, could contribute to an improved personalized therapy.

Tumors are not homogeneous, and the use of one cell line would not adequately reflect the properties of the disease. Therefore, three PCa cell lines, LNCap (lymph node metastasis), PC3 (bone metastasis) and DU-145 (brain metastasis), were used in this study to best represent tumor heterogeneity and disease progression having different proliferation rates, metastatic potential and rate of androgen-independence.

To model anti-RTK therapy we selected trastuzumab targeting HER2 extracellularly, 17-DMAG targeting Hsp90 intracellularly, or their combination. HER2 receptor expression level in the selected cell lines is 20,000-50,000 receptors/cell (27) which corresponds to +1 on a HercepTest (13). This is considered to be a low expression, as compared to $\mathrm{BCa}$ patients having a strong expression $(+3)$ and receiving anti-HER2 therapy. However, HER2 expression on the level of +1 is typical for PCa (13). It has also been taken in consideration that HER2 expression in $\mathrm{PCa}$ is associated with advanced androgen-independent disease and HER2 as a member of RTK-family is actively involved in the outlaw pathway of androgen-resistant PCa. Since HER2 expression in $\mathrm{PCa}$ is associated with the advanced disease stage, its expression cannot be derived from primary tumor biopsy material. Receptor expression at this stage could be detected using molecular imaging with PET or SPECT modalities. Detection of +1 expression in vivo is challenging, but it has previously been shown that by using highly sensitive and small protein-based imaging probes (e.g., anti-HER2 affibody molecules), it has become possible to visualize such low levels of HER2 expression (32-35).

$\mathrm{PCa}$ cells receiving trastuzumab treatment alone over a long period of time demonstrated a different pattern of response (Fig. 1) in spite of similar HER2 expression in these cell lines (27). The Hsp90 inhibitor 17-DMAG when used alone affected cell growth significantly in all tested cell lines. The combination of two treatments, did not result in an additive decrease in cell proliferation in LNCap cells, but contributed with an additive effect for PC 3 and DU-145 cells. We can conclude that anti-HER 2 treatment using trastuzumab alone did not result in a strong anti-proliferative effect in tested PCa cell lines. This is in agreement with therapy efficacy in $\mathrm{BCa}$ where treatment of +1 tumors with trastuzumab is ineffective. However, combination of trastuzumab and Hsp90 inhibition gave a negative effect on cell growth in all treated PCa cell lines.

After 8 weeks of treatment HER2 and EGFR expression of all cells lines were analyzed. We found that even though Hsp90 inhibitor clearly demonstrated a growth reducing effect in all cell lines, only cells strongly affected by this treatment responded with downregulation (LNCap) or stable (DU-145) HER2 expression. On the contrary, PC3 cells that showed a moderate response to 17-DMAG treatment demonstrated increased HER2 expression. According to the inhibiting function of 17-DMAG, i.e., prevention of the intracellular production of HER2 $(25,36)$, our results contradict literature data. However, previous studies were made on cell lines with high HER2 expression, and the receptor levels were estimated using western blot analysis, while we quantified the membranous expression. Our study set-up also differs by the drug exposure time, since we studied the receptors expression after 8 weeks of continuous treatment. We therefore suggest that cell lines such as PC3 with elevated HER2 expression post-treatment could be an indication of treatment resistance, whereas cell lines such as LNCap and DU-145 with increased EGFR expression and stable or downregulated HER2 expression respond well to the therapy modality. This observation could be used for therapy response monitoring.

During long-term treatment with trastuzumab/17-DMAG, upregulation of EGFR expression was observed in cell lines 
responding to all treatments (LNCap and DU-145 cells). This was especially pronounced in the combination of trastuzumab and 17-DMAG, which resulted in a significant EGFR expression increase in DU-145 cells (Fig. 2). On the contrary, PC3 demonstrated a small, but significant decrease in EGFR expression. EGFR is the preferable partner for HER2 heterodimerization and is also involved in the outlaw pathway of androgen-independent PCa. The significant upregulation of EGFR expression in cells responding to anti-HER2 therapy leads us to speculate on the possibility of using drugs to inhibit the action from HER2/EGFR complex formation by blockage of several signaling pathways simultaneously, hence inhibiting PCa cell proliferation. Monoclonal antibody cetuximab, which is used in treatment of EGFR expressing colorectal cancer (26), was used in combination with trastuzumab on our panel of PCa cells, since all cell lines express both EGFR and HER2 (27,28). The results from EGFR/HER2 targeted treatment demonstrated that growth of LNCap cells was strongly affected by sole cetuximab treatment as well as by the addition of trastuzumab, which also is in agreement with the elevated EGFR expression as response to trastuzumab treatment. PC3 cells remained unaffected (Fig. 3). DU-145 cells, although previously showing an increased EGFR expression, did not demonstrate any major difference in cell growth when exposed to the trastuzumab/cetuximab treatments. Moreover, there was no correlation between initial EGFR expression in tested cell lines and response to anti-EGFR therapy. LNCap cells having $\sim 40,000$ receptors/cells which is 2-fold lower than in PC3 cells and 5-fold lower than in DU-145 cells (28), strongly responded to anti-EGFR therapy, when other cells showed minor response. However, no additive effect from trastuzumab/cetuximab treatment was observed in any cell line.

This study is a preclinical evaluation using some of the already available treatments and addresses the fact that all treatments potentially change the RTK expression profile of the tumor. Such changes affect the output of the treatment and may also be used for therapy monitoring.

Simultaneous targeting of different receptors is already being used in clinics (37) and shows to be beneficial as compared to single-receptor targeting (38). Concerning PCa, 17-DMAG has been administered to patients with castrate resistant PCa (39), already on concomitant treatment, and the outcome was very positive, indicating that combination treatments could further improve the treatment of late stage of $\mathrm{PCa}$.

Our data suggest that simultaneous blocking of action of several receptors and factors involved in the proliferation of PCa cells can change the phenotypic profile of the tumor and treatment outcome could be monitored and possibly improved by observation of such changes. Understanding of molecular pathways, identification of biomarkers and proper patient stratification is crucial for the best outcome of targeted treatment, and we therefore suggest a further investigation into the significance of RTK expression for patient stratification and as response to RTK-targeting therapy.

\section{Acknowledgements}

This research was financially supported by Swedish Cancer Society (Cancerfonden) and the Swedish Research Council (Vetenskapsradet). The authors would like to gratefully acknowledge Apoteket Farmaci AB, Cytostatikaberedningen Uppsala for providing this research with trastuzumab and cetuximab, and Affibody AB, Solna for providing us with $\mathrm{Z}_{2395}$ affibody molecules.

\section{References}

1. The National Board of Health and Welfare (Socialstyrelsen): Nationella riktlinjer för bröst-, prostata-, tjocktarms- och ändtarmscancervård 2013. ISBN 978-91-7555-038-1, 2013-2-29 (in Swedish).

2. Sieh W, Lichtensztajn DY, Nelson DO, et al: Treatment and mortality in men with localized prostate cancer: a populationbased study in California. Open Prost Cancer J 6: 1-9, 2013.

3. Stattin P, Johansson R, Lodnert R, et al: Geographical variation in incidence of prostate cancer in Sweden. Scand J Urol Nephrol 39: 372-379, 2005.

4. Carter HB: American Urological Association (AUA) guideline on prostate cancer detection: process and rationale. BJU Int 112: 543-547, 2013.

5. Kelloff GJ, Choyke P, Coffey DS and Prostate Cancer Imaging Working Group: Challenges in clinical prostate cancer: role of imaging. Am J Roentgenol 192: 1455-1470, 2009.

6. Widmark A, Klepp O, Solberg A, et al: Endocrine treatment, with or without radiotherapy, in locally advanced prostate cancer (SPCG-7/SFUO-3): an open randomised phase III trial. Lancet 373: 301-308, 2009.

7. Tannock IF, de Wit R, Berry WR, et al: Docetaxel plus prednisone or mitoxantrone plus prednisone for advanced prostate cancer. New Engl J Med 351: 1502-1512, 2004.

8. Petrylak DP, Tangen CM, Hussain MH, et al: Docetaxel and estramustine compared with mitoxantrone and prednisone for advanced refractory prostate cancer. New Engl J Med 351: 1513-1520, 2004.

9. Pienta KJ and Bradley D: Mechanisms underlying the development of androgen-independent prostate cancer. Clin Cancer Res 12: 1665-1671, 2006.

10. Signoretti S, Montironi R, Manola J, et al: Her-2-neu expression and progression toward androgen independence in human prostate cancer. J Natl Cancer Inst 92: 1918-1925, 2000.

11. Craft N, Shostak Y, Carey M and Sawyers CL: A mechanism for hormone-independent prostate cancer through modulation of androgen receptor signaling by the HER-2/neu tyrosine kinase. Nat Med 5: 280-285, 1999.

12. Culig Z, Hobisch A, Cronauer MV, et al: Androgen receptor activation in prostatic tumor cell lines by insulin-like growth factor-I, keratinocyte growth factor, and epidermal growth factor. Cancer Res 54: 5474-5478, 1994.

13. Minner S, Jessen B, Stiedenroth L, et al: Low level HER2 overexpression is associated with rapid tumor cell proliferation and poor prognosis in prostate cancer. Clin Cancer Res 16: 1553-1560, 2010.

14. Yarden Y and Sliwkowski MX: Untangling the ErbB signalling network. Nat Rev Mol Cell Biol 2: 127-137, 2001.

15. So A, Gleave M, Hurtado-Col A and Nelson C: Mechanisms of the development of androgen independence in prostate cancer. World J Urol 23: 1-9, 2005.

16. Bacus SS, Altomare DA, Lyass L, et al: AKT2 is frequently upregulated in HER-2/neu-positive breast cancers and may contribute to tumor aggressiveness by enhancing cell survival. Oncogene 21: 3532-3540, 2002.

17. Hynes NE and Lane HA: ERBB receptors and cancer: the complexity of targeted inhibitors. Nat Rev Cancer 5: 341-354, 2005.

18. Vacchelli E, Aranda F, Eggermont A, et al: Trial Watch: Tumor-targeting monoclonal antibodies in cancer therapy. Oncoimmunology 3: e27048, 2014.

19. Sharma PS, Sharma R and Tyagi T: Receptor tyrosine kinase inhibitors as potent weapons in war against cancers. Curr Pharm Des 15: 758-776, 2009.

20. Altai M, Orlova A and Tolmachev V: Radiolabeled probes targeting tyrosine-kinase receptors for personalized medicine. Review invited. Curr Pharm Des 20: 2275-2292, 2014.

21. Albanell J, Codony J, Rovira A, Mellado B and Gascón P: Mechanism of action of anti-HER2 monoclonal antibodies: scientific update on trastuzumab and 2C4. Adv Exp Med Biol 532: 253-268, 2003. 
22. The US National Institutes of Health: Evaluation of carboplatin/ paclitaxel with and without trastuzumab (Herceptin) in uterine serous cancer. ClinicalTrials.gov, NCT01367002, 2014-2-13. http://clinicaltrials.gov/ct2/show/NCT01367002?term=NCT0136 $7002 \&$ rank $=1$.

23. The US National Institutes of Health: Safety study of ${ }^{212} \mathrm{~Pb}-\mathrm{TCMC}$-trastuzumab radio immunotherapy. ClinicalTrials gov, NCT01384253, 2014-2-13. http://clinicaltrials.gov/show/ NCT01384253.

24. The US National Institutes of Health: Paclitaxel and radiation therapy with or without trastuzumab in treating patients who have undergone surgery for bladder cancer. ClinicalTrials.gov, NCT00238420, 2014-2-13. http://clinicaltrials.gov/ct2/show/ study/NCT00238420.

25. Zuehlke A and Johnson JL: Hsp90 and co-chaperones twist the functions of diverse client proteins. Biopolymers 93: 211-217, 2010.

26. Wheeler DL, Huang S, Kruser TJ, et al: Mechanisms of acquired resistance to cetuximab: role of HER (ErbB) family members. Oncogene 27: 3944-3956, 2008.

27. Malmberg J, Tolmachev V and Orlova A: Imaging agents for in vivo molecular profiling of disseminated prostate cancer: Cellular processing of [(111)In]-labeled CHX-A"DTPAtrastuzumab and anti-HER 2 ABY-025 Affibody in prostate cancer cell lines. Exp Ther Med 2: 523-528, 2011.

28. Malmberg J, Tolmachev V and Orlova A: Imaging agents for in vivo molecular profiling of disseminated prostate cancer targeting EGFR receptors in prostate cancer: Comparison of cellular processing of [ [111 In]-labeled affibody molecule $\mathrm{Z}_{\text {EGFR:2377 }}$ and cetuximab. Int J Oncol 38: 1137-1143, 2011.

29. Ahlgren S, Orlova A, Wållberg H, et al: Targeting of HER2expressing tumors using 111 In-ABY-025, a second-generation affibody molecule with a fundamentally reengineered scaffold. J Nucl Med 51: 1131-1138, 2010.

30. Baek KH, Hong ME, Jung YY, et al: Correlation of AR, EGFR, and HER2 expression levels in prostate cancer: Immunohistochemical analysis and chromogenic in situ hybridization. Cancer Res Treat 44: 50-56, 2012.
31. Carrion-Salip D, Panosa C, Menendez JA, et al: Androgenindependent prostate cancer cells circumvent EGFR inhibition by overexpression of alternative HER receptors and ligands. Int J Oncol 41: 1128-1138, 2012.

32. Malmberg J, Perols A, Varasteh Z, et al: Comparative evaluation of synthetic anti-HER2 Affibody molecules sitespecifically labelled with ${ }^{111}$ In using N-terminal DOTA, NOTA and NODAGA chelators in mice bearing prostate cancer xenografts. Eur J Nucl Med Mol Imaging 39: 481-492, 2012.

33. Wållberg H, Orlova A, Altai M, et al: Molecular design and optimization of $99 \mathrm{mTc}$-labeled recombinant affibody molecules improves their biodistribution and imaging properties. J Nucl Med 52: 461-469, 2011.

34. Baum RP, Prasad V, Müller D, et al: Molecular imaging of HER2-expressing malignant tumors in breast cancer patients using synthetic ${ }^{111} \mathrm{In}$ - or 68Ga-labeled Affibody molecules. J Nucl Med 51: 892-897, 2010.

35. Sörensen J, Sandberg D, Sandström M, et al: First-in-human molecular imaging of HER2 expression in breast cancer metastases using the ${ }^{111}$ In-ABY-025 Affibody molecule. J Nucl Med 55: 730-735, 2014.

36. Kramer-Marek G, Kiesewetter DO and Capala J: Changes in HER 2 expression in breast cancer xenografts after therapy can be quantified using PET and (18)F-labeled affibody molecules. J Nucl Med 50: 1131-1139, 2009.

37. Clinic H, Hallwang Clinic Treatments. 2014-2-13. http://www. germancancertreatments.com/treatments-at-hallwang-clinic/.

38. Andersson K: Bringing time into molecular and cellular biology. J Anal Oncol 2: 65, 2013.

39. Pacey S, Wilson RH, Walton M, et al: A phase I study of the heat shock protein 90 inhibitor alvespimycin (17-DMAG) given intravenously to patients with advanced solid tumors. Clin Cancer Res 17: 1561-1570, 2011 